\title{
从物理视角看花岗质岩浆在非运移过程中的 结晶分异
}

陈晨 ${ }^{1,2 *}$, 丁兴 $3,7 \dagger$, 李寊 ${ }^{2,4}$, 张维骐 2,4 , 欧阳东剑 2,4 , 杨雷 2,4 , 孙卫东 $5,6,7$

1. 中国科学院广州地球化学研究所中国科学院矿物学与成矿学重点实验室, 广州 510640;

2. 中国科学院大学, 北京 100049 ;

3. 中国科学院广州地球化学研究所同位素地球化学国家重点实验室, 广州 510640;

4. 中国科学院地质与地球物理研究所岩石圈演化国家重点实验室, 北京 100029 ;

5. 中国科学院海洋研究所深海研究中心, 青岛 266071;

6. 青岛海洋科学与技术国家实验室海洋矿产资源实验室, 青岛 266237;

7. 中国科学院青藏高原地球科学卓越创新中心, 北京 100101

*通讯作者, E-mail: chencchina@gmail.com

†通讯作者, E-mail: xding@gig.ac.cn

收稿日期: 2016-10-29; 接受日期: 2017-10-09; 网络版发表日期: 2017-12-26

国家科技部重点研发计划项目(编号: 2016YFC0600204、2016YFC0600408)、国家自然科学基金项目(批准号: 41421062、41372005)资助

摘要花岗岩是地球区别于太阳系其他行星的重要特征, 研究花岗岩的演化对于理解现今地球大陆地壳的 形成有重要意义. 结晶分异是岩浆演化的主要机制之一。然而, 由于花岗质岩浆黏度高, 为非牛顿流体, 结晶分 异在酸性岩浆中有效与否仍有争议. 本文侧重物理分析方法, 以此审视花岗质岩浆在非运移过程——在岩浆房 中及岩浆就位后的结晶分异作用。通过物理计算及分析, 我们认为, 花岗质岩浆高稆度的特性使得一般的矿物 颗粒在岩浆房中受阻沉降速度极小 $\left(\sim 10^{-9} \sim 10^{-7} \mathrm{~m} \mathrm{~s}^{-1}\right)$, 因而在存在岩浆对流时, 颗粒的堆晶过程将受到影响, 岩 浆成分趋于均一; 当岩浆房演化至晶粥状态(结晶度F> 40 50\%)后, 岩浆对流基本停止, 此时粒间熔体可通过颗 粒的受阻沉降及压实作用挤出, 汇聚成高硅熔体层. 高硅熔体层可进一步形成高硅花岗岩、流纹岩. 在岩浆房 演化至不同程度时, 晶粥体多期次的活化及岩浆的上侵可能形成成分变化的复式岩体. 此外, 以华南富锂氟花 岗岩为代表的特殊花岗岩类, 相对于一般花岗质岩浆具有更低的黏度和固相线, 可能以结晶分异作用产生矿物 组合及成分上的垂向分带。而侵入体中小尺度的成分变化结构不是重力分异的结果, 流动分异或许起着关键 作用. 综合来说, 花岗质岩浆能够发生结晶分异; 高分异特征的高硅花岗岩及火山岩可能是酸性岩浆结晶分异 的产物, 而花岗岩可能是结晶分异形成的堆晶.

关键词花岗岩, 结晶分异, 岩浆对流, 层状构造, Mush模型, 高分异花岗岩, 复式岩体, 富锂氟花岗岩

中文引用格式: 陈晨, 丁兴, 李睿, 张维骐, 欧阳东剑, 杨雷, 孙卫东. 2018. 从物理视角看花岗质岩浆在非运移过程中的结晶分异. 中国科学: 地球科学, 48: 261-275, doi: 10.1360/N072016-00355

英文引用格式： Chen C, Ding X, Li R, Zhang W Q, Ouyang D J, Yang L, Sun W D. 2018. Crystal fractionation of granitic magma during its non-transport processes: A physics-based perspective. Science China Earth Sciences, 61: 190-204, https://doi.org/10.1007/s11430-016-9120-y 


\section{1 引言}

花岗岩是地球大陆地壳的重要组成部分, 是我们星 球在太阳系中区别于其他行星的重要特征(Campbell 和 Taylor, 1983; Taylor和 McLennan, 1985; 吴福元等, 2007), 因而花岗质岩浆成因及演化的研究一直备受关 注. 已有的研究显示, 结晶分异在岩浆演化过程中扮演 了重要的角色, 是岩浆成分分异的主要机制之一(Wager 和Brown, 1968; Lee和Bachmann, 2014). 岩浆结晶时, 晶出的矿物与岩浆成分不同; 如果矿物与熔体分离, 那 么岩浆的成分将发生改变, 结晶分异以此使岩浆产生 分异(Becker, 1897; 吴福元等, 2017). 根据作用机理的 不同, 前人提出了多种结晶分异的具体机制, 主要包 括受阻沉降(hindered settling, Davis和Acrivos, 1985)、 晶体压实作用(compaction, McKenzie, 1984, 1985)、对 流分异(convective fractionation, Sparks等, 1984)和流动 分异(flowage differentiation, Bhattacharji和Smith, 1964; Barrière, 1976).

对以上几类结晶分异机制的认识主要来源于对 镁铁质岩浆的研究, 然而, 对于酸性岩浆能否有效地 发生结晶分异作用目前仍存有较大争议. 由于花岗质 岩浆较玄武质岩浆更为富 $\mathrm{Si}$, 二者黏度存有数量级的 差别, 一些学者对花岗质岩浆发生结晶分异的可能性 提出了质疑(Reid等, 1993 ; Pitcher, 1997; 张旗等, 2007; 张旗, 2012; Clemens等, 2010; Clemens和Stevens, 2012; Gualda等, 2012). 比如, 张旗等(2007)和张旗(2012)指 出, 花岗质岩浆高的黏度会阻碍晶体生长且妨碍矿物 沉降, 而主要造岩矿物和花岗质岩浆之间密度差较小, 使得结晶分异难以进行. 在野外, 玄武质和安山质岩 石往往伴生有相应的堆晶岩, 而与花岗岩体相关的堆 晶岩则少有被发现, 这从另外一个角度似乎说明花岗 质岩浆发生结晶分异的可能性很小(张旗等, 2007). 然 而, 需要指出的是, 酸性岩浆与镁铁质-超镁铁质岩浆 这种黏性上的巨大差异, 可能导致二者结晶分异产生 的“堆晶”在表现形式上有着显著区别, 中-基性岩堆晶 的野外特征能否用以判别花岗质岩浆的堆晶存有疑 问.

与之同时, 哪些花岗岩体中的地质现象与结晶分 异有关? 学者们对此也持有不同的见解. 例如, 岩体中
的层状构造、钾长石巨晶“堆晶”结构, 这些在小尺度 上成分变化的结构是否是堆晶作用的产物就极具争议 (例如, Clarke和Clarke, 1998; Glazner等, 2008; Streck和 Grunder, 2008; Solgadi和Sawyer, 2008); 而一些大型岩 体较大的成分变化是否由结晶分异引起同为争议的 焦点(Bateman和Chappell, 1979; Tindle和Pearce, 1981; Coleman等, 2004; Glazner等, 2004). 因此, 寻找确切 的岩石学证据是佐证花岗质岩浆可以结晶分异的关 键. 近来, 有研究表明, 火山喷出岩与侵入体似乎有着 密切的成因联系(Hildreth, 2004; Bachmann和Bergantz, 2004; Lundstrom和 Glazner, 2016), 流纹岩和高硅花岗 岩很可能是花岗质岩浆结晶分异的产物(Bachmann等, 2007; Lee和Morton, 2015; Lee等, 2015). 此外,一些特 殊的花岗岩类(如富锂氟花岗岩)常常与矿床成因有关, 而在野外有时可以看到其与低分异花岗岩之间的共 生关系(Yin等, 1995; Zhu等, 2001). 这为理解花岗质岩 浆结晶分异作用提供了更多路径.

本文根据岩浆的汇聚状态, 将岩浆演化划分为岩 浆运移过程和非运移过程. 岩浆运移指岩浆产生后, 在从源区到岩浆房以及从岩浆房到岩浆就位位置等 过程中需要通过某种岩浆通道进行运输的过程; 而非 运移过程则包括岩浆房过程及岩浆从岩浆房上侵就 位后逐渐冷却结晶的过程. 显然, 岩浆在这两类过程 中经历的物理过程不同. 前者涉及通道中的岩浆流动, 而后者则更为复杂, 通常情况下对最终产生的花岗质 岩石的成分起决定作用. 为方便论述, 本文仅涉及一 般研究中更为关注的岩浆非运移过程. 而在分析这一 过程时, 运移过程中岩浆分异产生的干扰则需剔除. 对于花岗质岩浆结晶分异问题, 前人更多的是从地球 化学及野外地质角度反演分析, 其解译存在着多解(张 旗等, 2007). 更为关键的是, 一定结晶度的花岗质岩 浆为非牛顿流体, 具有高的黏度, 这是致使学者们对 其结晶分异作用产生质疑的根源(马昌前, 1989; 张旗 等, 2007). 因此, 本文选取物理视角来审视花岗质岩 浆非运移过程中的结晶分异作用, 主要试图回答两个 问题: (1) 花岗质岩浆能否发生结晶分异作用? (2) 若 能, 哪些地质现象与花岗质岩浆结晶分异有关? 花岗 质岩浆结晶分异过程中的关键物理因素以及相关地 质现象与结晶分异的联系都将在下文进行讨论. 


\section{2 花岗质岩浆房及其中矿物行为的物理特} 性

\section{1 晶体颗粒在牛顿流体与酸性岩浆(非牛顿流 体)中的沉降行为}

结晶矿物能否相对富集、能否与岩浆产生相对 位移是结晶分异作用有效进行与否的关键(吴福元等, 2017). 因而, 了解矿物晶体在岩浆中的行为对于理解 结晶分异的机制至关重要. 晶体颗粒在岩浆中的运动 与岩浆的黏度、结晶度、流动状态等因素密切相关. 其中, 黏度主要受岩浆成分及温度控制, 它是玄武质岩 浆和花岗质岩浆存在显著差异的物理性质之一. 通常 来说, 玄武质和花岗质岩浆的黏度相差巨大. 例如, 富 $\mathrm{H}_{2} \mathrm{O}$ 、贫晶体的高硅岩浆, 其黏度 $10^{4.5} \mathrm{~Pa}$ s(Scaillet等, 1998; Clemens和Petford, 1999), 而经历一定分异演化 的玄武质岩浆其黏度为 $10^{2} \mathrm{~Pa}$ s(Murase and McBirney, 1973), 二者相差近 3 个数量级. 而富 $\mathrm{Si}$ 、富 $\mathrm{H}_{2} \mathrm{O}$ 的高黏 度熔体的形成与岩浆的结晶演化有关. 如安山质和 英安质岩浆 $\left(\mathrm{SiO}_{2}=60 \sim 70 \mathrm{wt} \%\right)$ 冷凝固结过程中, 由于斑 晶矿物的大量结晶, 导致剩余熔体(即对应岩石的基 质玻璃)演化为高硅流纹质的成分(Maughan等, 2002; Sparks等, 2008). 因此, 我们有必要了解花岗岩造岩矿 物在酸性岩浆中的行为特征.
晶体颗粒在牛顿流体和非牛顿流体体系下将遵 循不同的物理规律 (马昌前, 1987, 1989). 简单来说, 牛 顿流体是指任一点上的剪应力都同剪切变形速率呈 线性函数关系的流体, 不满足此条件的流体可统称为 非牛顿流体; 非牛顿流体要产生形变需克服屈服力(马 昌前, 1987, 1989; Street等, 1996). 前人研究发现, 结 晶度对熔体的流体性质将产生巨大影响. 在Martin和 Nokes(1988)的实验研究中, 他们观察到, 在颗粒质量 分数 $<0.01 \mathrm{wt} \%$ 的流体体系中, 晶体的沉降行为满足牛 顿流体下的运动规律. 而后Koyaguchi等(1990)对一较 Martin和Nokes(1988)研究体系略微浓稠的体系(颗粒 $0.3 \mathrm{wt} \%, 0.1 \mathrm{vol} . \%)$ 中颗粒的运动进行观察. 通过观测 他们发现, 该体系中颗粒的运动行为发生了显著变化, 流体表现出非牛顿流体性质.

在颗粒质量分数 $<0.01 \mathrm{wt} \%$ 的静态牛顿流体体系 中, 对其中的晶体颗粒(本文讨论的晶体颗粒均假定为 球体)进行简单的受力分析可知(图1), 晶体颗粒静止 时, 将受到重力与浮力作用. 若重力大于或小于浮力, 颗粒将下沉或上浮. 随后, 晶体开始运动, 岩浆施予拖 拽力. 拖拽力与颗粒运动方向相反, 力的大小与颗粒 运动速度 $(U)$ 成正相关. 颗粒将做变加速运动, 直到速 度增加至晶体受力达平衡, 此后颗粒保持匀速运动. 其中, Stokes 拖拽力 $\left(F_{\text {Stokes }}\right)$ 为

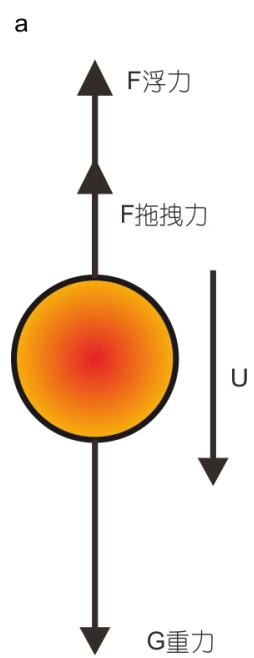

b

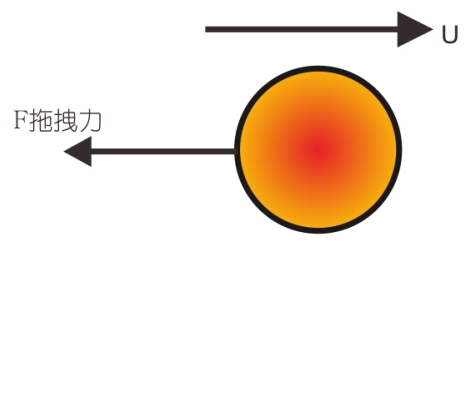

c

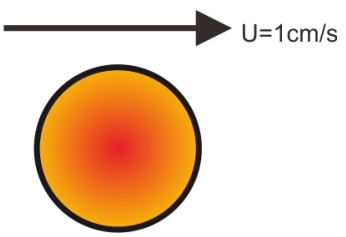

$2 \mu s$ 后

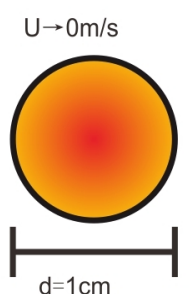

相对位移 $5 \mathrm{~nm}$

图 1 岩浆中矿物颗粒运动及受力示意图

(a) 静态岩浆中, 密度大于岩浆的矿物受阻沉降时的受力分析示意图; (b) 运动的矿物颗粒在岩浆中的受力分析图解(忽略重力); (c) 给一粒 径为 $1 \mathrm{~cm}$ 的长石或石英颗粒相对岩浆 $1 \mathrm{~cm} \mathrm{~s}^{-1}$ 的速度, 在流纹质岩浆中, 颗粒将在 $2 \mu \mathrm{s}$ 后趋于静止, 和岩浆产生的相对位移为矿物半径的百万 分之一. 因而可知, 岩浆紊乱流动时, 矿物将被岩浆裹挟运动而难以因重力下沉 


$$
F_{\text {Stokes }}=-6 \pi \mu r U
$$

(Street等, 1996), 根据颗粒受力平衡得 $U_{\text {Stokes }}$ 斯托克斯 沉降速度：

$$
U_{\text {Stokes }}=\frac{2 r^{2} \mathrm{~g} \Delta \rho}{9 \mu},
$$

式中, $r$ 为晶体颗粒半径, $\mathrm{g}$ 为重力加速度, $\Delta \rho$ 为晶体与 熔体的密度差, $\mu$ 代表熔体的动态黏度. 假设一个半 径为 $2 \mathrm{~mm}$ 的石英或长石颗粒 (密度约为 $2600 \mathrm{~kg} \mathrm{~m}^{-3}$ ), 在 晶体质量分数 $<0.01 \mathrm{wt} \%$ 、密度 $2300 \mathrm{~kg} \mathrm{~m}^{-3}$ 、黏度为 $10^{4.5} \mathrm{~Pa} \mathrm{~s}$ 的流纹质熔体中下沉, 计算可知, 颗粒下沉速 度为 $8.43 \times 10^{-8} \mathrm{~m} \mathrm{~s}^{-1}$ (即 $2.66 \mathrm{~m} \mathrm{a}^{-1}$ ).

对于静态非稀悬浮液(non-dilute suspension, 颗粒 $>1 \mathrm{vol} . \%$ )的单分散性体系, 由于其存在屈服力, 我们可 用修正系数对颗粒的沉降速度进行矫正. 沉降速度可 表示为

$$
U_{\text {hs }}=U_{\text {stokes }} \cdot f(c),
$$

式中, $U_{\mathrm{hs}}$ 为沉降速度.

$$
f(c)=\frac{(1-c)^{2}}{\left(1+c^{1 / 3}\right)^{[5 c / 3(1-c)]}},
$$

式中, $f(c)$ 为修正系数, $c$ 为晶体分数 (Barnea和Mizrahi, 1973). 从图 2 可知, 随着结晶度的增加, $f(c)$ 减小, $U_{\mathrm{hs}}$ 急 剧下降. 结晶度大于 0.6 时, 修正系数已小于 $3.47 \times 10^{-2}$, 颗粒趋于静止. 单分散性系统的沉降速度公式提供 了颗粒沉降速度与岩浆结晶度的大体关系, 多分散 性体系更切合岩浆的实际情况, 但多分散体系下的颗 粒沉降行为更为复杂, 目前知之甚少(Davis和Acrivos, 1985).

通过式(2) (4)可知, 对于晶体沉降, 最重要的物 理影响因素是晶体颗粒半径及岩浆黏度. 式中 $r$ 可 在微米-厘米级别变化, 且平方放大了半径对速度的 影响, 而不同组分的岩浆黏度差别可达 13 个数量级 $\left(10^{1} \sim 10^{14} \mathrm{~Pa}\right.$ s, Murase和McBirney, 1973); 相较之下, 矿 物和岩浆的密度差一般为数百 $\mathrm{kg} \mathrm{m}^{-3}$, 为同一量级, 可 知, 密度差对沉降速度影响相对较小.

以上分析可知,颗粒 $>1 \mathrm{vol} \%$ 时, 流纹质熔体表现 为非牛顿流体, 同时黏度极大. 即便在这样的体系下, 晶体颗粒依旧可发生显著重力沉降, 前提是有足够的 沉降时间且岩浆房保持相对静态. 但重力分异的效应 还与岩浆房形状、岩浆房寿命、岩浆对流状态、矿物

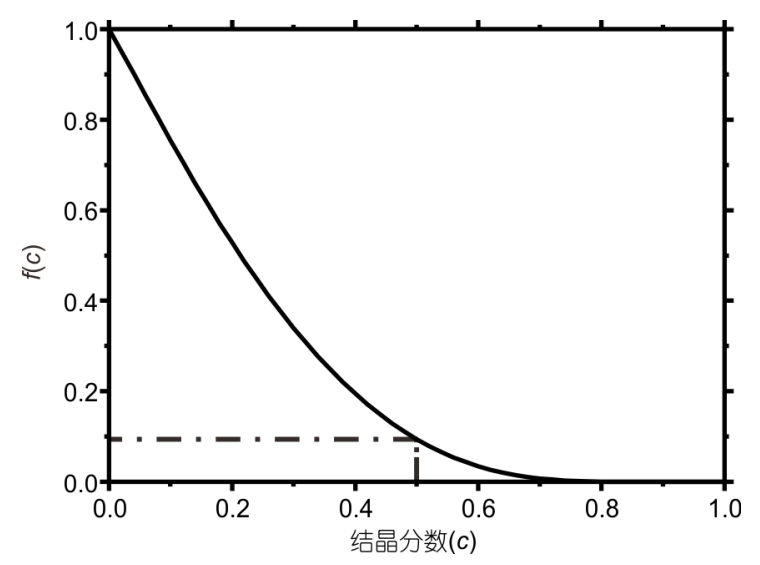

图 2 经验修正系数 $f(c)$ 随晶体分数 $(c)$ 的演化图解 由图可知, 当晶体分数 (c) 增加, 晶体沉降速度 $U_{\mathrm{hs}}=U_{\text {Stokes }} \cdot f(c)$ (Barnea 和Mizrahi, 1973) 将急剧下降. 在 $c$ 值为 0.5 时沉降速度将下降约一个 量级. 据Bachmann和Bergantz(2004)修改

结晶序列、不同矿物间沉降速度差等诸多因素有关. 因此, 不能简单地将花岗质岩浆的结晶分异过程与玄 武质岩浆等同分析. 值得注意的是, 上述体系所附带的 前提一一静态岩浆房. 通过式(3)计算可知, 当结晶度 为 0.5 时, 石英或长石颗粒的下沉速度约为 $7.961 \times 10^{-9} \mathrm{~m}$ $\mathrm{s}^{-1}$ (即 $0.251 \mathrm{~m} \mathrm{a}^{-1}$ ). 在如此小的沉降速度下, 微弱的岩 浆对流都可能对晶体的沉降产生巨大扰动. 因而, 由 重力驱动矿物与熔体分离, 就需要岩浆保持静态. 大 多情况下, 岩浆房的对流状态及晶体在流动岩浆中的 运动特性, 对岩浆房中晶体的散布有着决定性影响.

\section{2 晶体在流动的黏滞岩浆中的行为特征}

如上文所述, 晶体在岩浆中的运动学行为与重 力、浮力、运动时岩浆产生的拖拽力等因素有关; 除 此之外, 岩浆的流动状态对颗粒的运动同样关键. 我 们借助Glazner(2014)的相关计算来探讨晶体颗粒在中 酸性岩浆中的行为特点.

假定给一中酸性岩浆中的球状晶体颗粒施加推 力, 当颗粒达到速度 $U$ 后立即撤去推力. 受力分析可 知, 在忽略重力作用的情况下, 晶体将在岩浆的拖拽 力作用下减速, 直到运动停止(图1c). 由式(1)及牛顿 第二定律可得

$$
F=-6 \pi \mu r U=m a .
$$

根据加速度的微积分含义可得到 


$$
a=k U=\frac{\mathrm{d} U}{\mathrm{~d} t}
$$

此处 $k=-6 \pi \mu r / m$, 对式(6)进行积分有

$$
U=U_{0} \mathrm{e}^{k t}
$$

式中, $U_{0}$ 为初始速度, $t=-k^{-1}$, 通过下式可得晶体的滑 行距离

$$
d=\int_{0}^{\infty} U \mathrm{~d} t=U_{0} t .
$$

假定我们给一直径为 $1 \mathrm{~cm}$ 的石英颗粒施加推力, 使其速度达到 $1 \mathrm{~cm} \mathrm{~s}^{-1}$, 之后将推力撤去. 根据上述推 导的公式计算可知, 颗粒的滑行距离 $d=5 \mathrm{~nm}$ (为该石英 颗粒半径的百万分之一), 并且该石英颗粒会在 $2 \mu \mathrm{s}$ 内 降至初始速度的 1\%(Glazner, 2014). 该计算结果为理 解晶体在硅酸盐岩浆中的运动提供了非常关键的信 息, 即岩浆以较大流速(相较于 2.1 章节计算中的受阻 沉降速度)流动时, 晶体颗粒与熔体几乎不产生相对位 移. 也就是说, 当岩浆流动时, 晶体颗粒来不及受重力 驱动向下沉降, 便被岩浆裹挟一同参与对流.

\section{3 岩浆房各演化阶段的岩浆对流}

如前所述, 晶体在静态的岩浆房中沉降速度十 分微弱, 仅 $10^{-9} \mathrm{~m} \mathrm{~s}^{-1}$ 数量级, 而岩浆对流流速可达 $\sim 0.01 \sim 0.1 \mathrm{~m} \mathrm{~s}^{-1}$ (Sparks等, 1984); 流纹质岩浆高的黏度 使得颗粒难以与流动的岩浆产生相对滑动. 因而, 岩浆 对流存在时, 晶体的分布主要受控于岩浆的对流行为.

尽管部分研究认为矿物结晶潜能热的释放使得 岩浆房热梯度小, 致使岩浆对流难以触发(Brandeis和 Marsh, 1989; Marsh, 1989a, 1989b, 1996), 但多数学者 的研究支持在矿物大量结晶之前的岩浆房早期阶段, 存在对流过程(马昌前等, 1994; Bachmann和Bergantz, 2008a; Huber等, 2009; Bea, 2010; Gutiérrez等, 2013). 对 于封闭体系下的花岗质岩浆房, 岩浆对流主要可分为 两个阶段, 即晶弹体前阶段和晶粥体阶段(Bea, 2010; Bachmann和Bergantz, 2004). 结晶度是划分对流阶段 的主要依据. 一般认为, 结晶度低于 0.5 时, 岩浆房内存 在对流; 而结晶度大于临界点( 0.5)时, 岩浆房将以晶 粥体(Mush)形式存在, 岩浆对流能力丧失(马昌前等, 1994; Bachmann和Bergantz, 2004; Bea, 2010). 在此章 节, 我们主要讨论结晶度 $<0.5$ 时, 岩浆房存在对流的过 程, 而晶粥体阶段的岩浆分异行为将在下文介绍讨论.
在岩浆房结晶度小于 0.5 时, 由于热梯度和负重力 梯度, 岩浆产生对流(Grout,1918), 这促使岩浆成分在 一定程度上更加均一(Whitney和Stormer,1985; Oldenburg等, 1989; Lindsay等, 2001; Christiansen, 2005). 也 一些学者认为对流使得岩浆有形成分层的趋势(Chen 和 Turner, 1980; McBirney, 1980; McBirney等，1985; Bergantz 和 Ni，1999; Jellinek 和 Kerr,1999; Jellinek 等, 1999). 在岩浆房边部及顶部的结晶前锋区域, 由于 与围岩接触, 冷却速率快, 较岩浆房其他部位结晶度更 高, 更早达到晶粥状态(Mush). 与此同时, Mush部分密 度增大, 形成负重力梯度. 当重力失稳时, 更为黏稠的 结晶前锋及边缘发生沉降, 引发对流. 随后, 周边结晶 度更低、温度更高的岩浆上浮填补匀出的空间. 周而 复始, 直至岩浆房冷却至结晶前锋不足以触发重力失 稳. 因此, 重力失稳引发的对流不可能使岩浆完全均 一. 若假定岩浆没有物质扩散, 那么先下沉的部分仅 比后填充至前锋区的岩浆更早达到更高的结晶度, 当 岩浆整体固结时, 其成分应不存在差别. 真实情况中 岩浆存在物质的交换, 但考虑到花岗质岩浆极大的黏 度 $\left(>10^{4} \mathrm{~Pa} \mathrm{~s}\right)$, 在没有进行对流混合时其物质交换是 有限的; 且结晶前锋物质在重力失稳沉降过程中与主 体岩浆会有较大空间、时间尺度的接触, 在此过程中 沉降的物质与主体岩浆成分上应趋于一致. 因此, 我 们认为对流使岩浆房具规律性成分变化的能力有限. Huber等(2009)对岩浆房对流的模拟计算结果表明, 在 岩浆房对流前期, 岩浆将混合均一; 对于岩浆对流逐渐 弱化、均一能力消减的阶段, 他们提出持续晶粥态作 用(mushification), 可促进岩浆房成分均一. 同时, 高温 岩浆的底侵可使晶粥体活化, 使岩浆房恢复对流, 岩浆 成分进一步趋同. 最后, 我们注意到许多花岗岩表现 为成分单一的中酸性岩体(Monotonous Intermediates, MI; Hildreth, 1981), 其在成岩温度、结晶度和主量成 分上均表现出显著的均一性(Bateman和Chappell,1979; Whitney和Stormer, 1985; Bachmann等, 2002). 而复式 岩体中单一相带的成分也多表现为MI. 因而, 我们倾 向于认为, 岩浆对流促使岩浆房成分均一; 同时, 在晶 粥体前阶段, 岩浆对流的存在使得晶体难以沉降, 熔 体与结晶矿物无法有效分离.

\section{4 花岗质岩浆房的活动寿命}

花岗质岩浆房的活动寿命决定了岩浆是否有充足 
的时间经历结晶分异以完成演化. 岩浆房寿命的长短 取决于岩浆体系热量的增、减. 热量的散失与岩浆对 流、热传导、围岩热物理性质等因素有关; 而热增量 主要来源于高温岩浆的补给和晶体结晶时的潜热释 放(Gelman等, 2013; Marsh, 1981). 在此, 我们不拟对岩 浆房的寿命问题进行详尽讨论, Bachmann(2011)对该 问题已有非常综合的论述. 众多的研究表明, 地壳中 的岩浆房固化速率极快, 小体量侵入体在几千年内温 度就能降至固相线以下, 而大型侵入体固化时间也不 会超过几十万年, 岩浆房的寿命尺度 $10^{3} \sim 10^{5} \mathrm{a}$ (Pitcher, 1997; Petford等, 2000; Glazner等, 2004; Stimac等, 2001; Cooper和Kent, 2014). 如Lee等 (2015)对一厚 $5 \mathrm{~km}$ 扁平 状侵入体的热演化模拟结果显示, 即便是侵位于中地 壳(地温背景温度 $400^{\circ} \mathrm{C}$ )、忽略岩浆对流影响的条件 下, 岩浆房仍将在 $1 \mathrm{Myr}$ 内降到锆石 Zr饱和温度及岩体 固相线温度以下(图3). 基于前人的研究, 我们将岩浆 房可记录的锆石年龄跨度基本限定为 $1 \mathrm{Myr}$.

\section{3 结晶分异作用解译花岗岩体中地质现象 的适用性}

\section{1 侵入体中的“假沉积构造”}

在了解矿物晶体在花岗质岩浆中的运动特性后, 我们不妨重新审视花岗岩体中的一些地质现象. 这 些现象通常被认为与岩浆结晶分异作用相关. 如

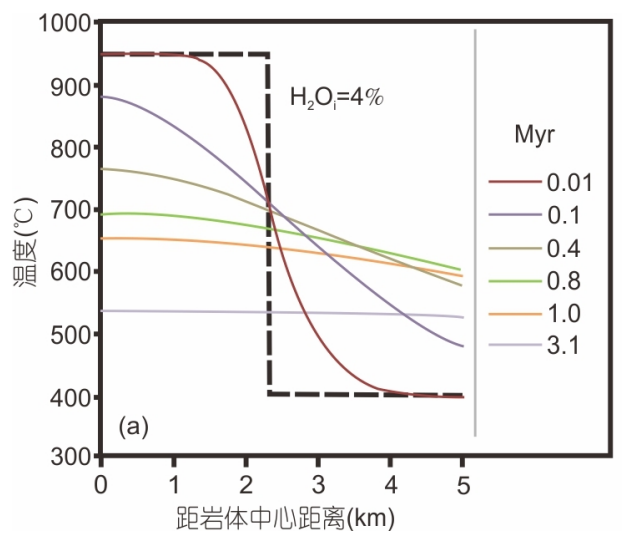

在花岗质岩体中常可观察到的层状结构 (图 $4 a$ 和 $b$ ), 其多在岩体边缘产出. 在传统观点中, 对于这类层 状结构, 一直以来习惯于和沉积侵蚀、晶体沉淀相 类比(例如, Gilbert, 1906; Emeleus, 1963; Smith, 1975; Clarke和 Clarke, 1998; Solgadi 和 Sawyer,2008; Barbey, 2009; Gaweda和Szopa, 2011), 也有研究认为这类结构 是流动分异剪切应力作用的结果(例如, Wilshire, 1969; Barrière, 1976; Bateman, 1992). 但如上文所述, 当岩浆 流动时, 晶体颗粒将与岩浆协同运动, 不能脱离岩浆 发生重力沉降. 岩浆流的扰动并不能使晶体通过重力 分选形成这类层理(Glazner, 2014), 这是由花岗质岩浆 高黏度的特点决定的.

虽然岩体中的层状构造无法完全类比沉积层理, 但这尚无法否定对流和压实在岩浆分异中的作用 (Clemens, 2015). 如图4c, 岩浆房各层位存在流速差 时, 会触发形成剪切力, 晶体在剪切力作用下可能形 成这类“卷轴状构造”. 受力分析可知, 在存在流速差 的岩浆中, 矿物受到的剪切力始终垂直于流速方向, 且剪切力始终与流速差共存, 可为一持续作用力, 不 受岩浆流扰动. 用流动分异来解释“卷轴状构造”的形 成, 与理论分析并不矛盾, 是合理的成因假说. 因此, 我们认为, “卷轴状构造”是晶体局部富集的产物, 应 该是花岗质岩浆流动分异的结果.

理论上看,一些构造现象虽然可以通过结晶分异 形成, 但深入研究发现, 这些结论可能还存有一定的

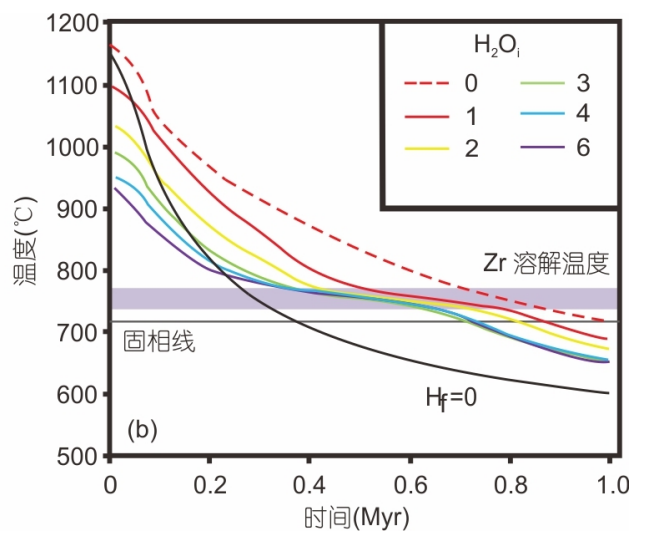

图 3 花岗质岩浆房的活动寿命示例图

(a) 一厚 $5 \mathrm{~km}$ 的扁平状岩体侵入到背景温度为 $400^{\circ} \mathrm{C}$ 的围岩中的热演化图解. 岩浆初始的温度假定与其液相线温度对应, 岩浆成分假设为含 $4 \mathrm{wt} \%$ 水的英云闪长质熔体. 图中展示了不同时间下的温度剖面. 热模拟考虑了晶体结晶潜热释放效应下的热传导, 但岩浆对流因素未考 虑在内. 虚线代表初始情况. (b) 体系水总含量不同时(假设为一封闭体系), 岩浆房中心温度与时间的函数关系. 岩浆初始温度与各水含量 下的液相线温度对应. 不同颜色的线代表不同水含量的体系的模拟情况. 所有模拟都假定总结晶潜热释放量为一定值. 黑色曲线代表总 结晶潜热为 0 的非实际情况. 据Lee等(2015)修改 

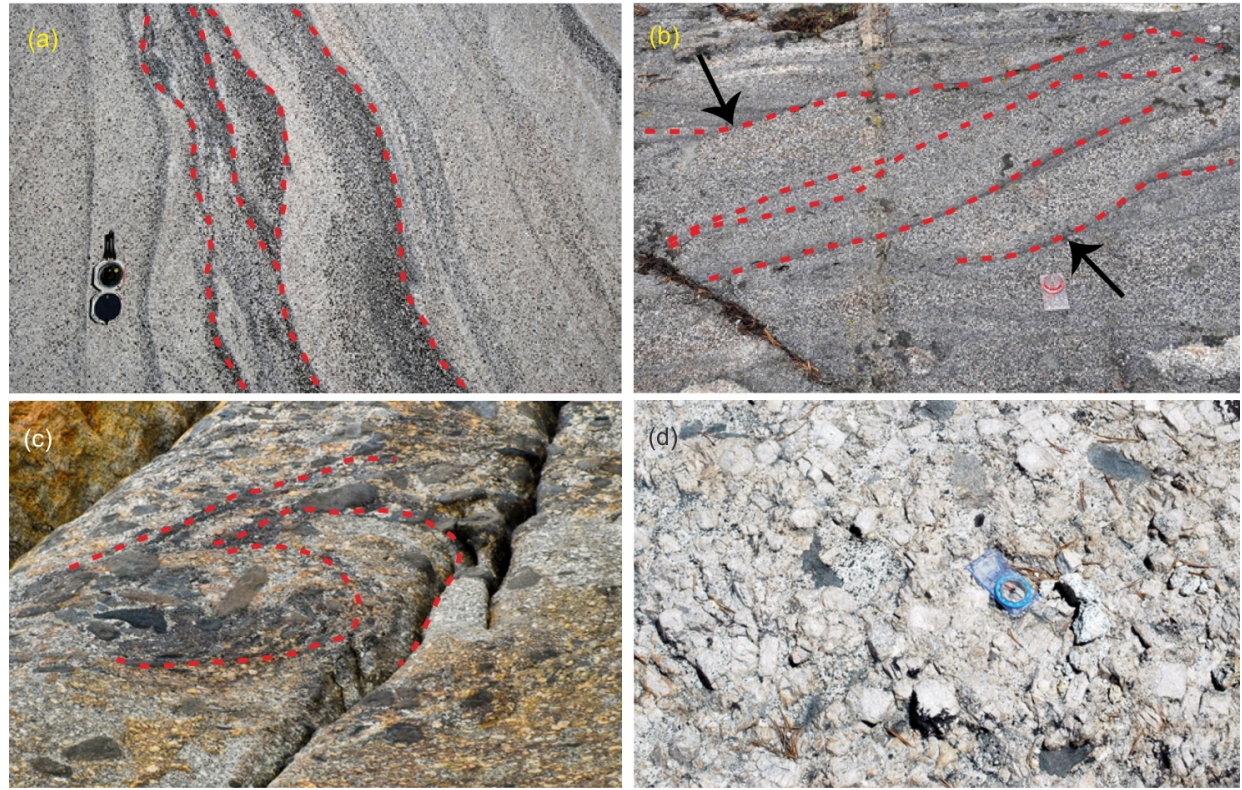

图 4 花岗岩体中的野外地质现象

(a) 花岗闪长岩中的“假沉积”构造, Half Dome花岗闪长岩, 加利福尼亚(美国)Tenaya Lake. 罗盘7cm宽. (图片来自 Glazner, 2014); (b) 截断层状 构造, 白严纪花岗闪长岩, 拍自加利福尼亚内华达山脉东部Rock Creek. 罗盘的长边为 $9.5 \mathrm{~cm}$; (c) 卷轴状构造, 可见巨钾长石斑晶、延展变 形的包体及云母、石榴子石、长石等矿物在局部富集. 发育于澳大利亚威尔逊岬的S型斑状二长花岗岩(Wallis, 1981). (图片来自Clemens, 2015); (d) 花岗闪长岩中的钾长石巨晶富集现象. 发育于美国加利福尼亚Yosemite国家公园. 巨晶长 5 10cm, 且占出露面积的 60 80\%. 罗盘 直径长 $5 \mathrm{~cm}$. (图片来自 Glazner, 2015)

疏漏. 例如, 图4d为美国加利福尼亚Yosemite国家公园 中发现的钾长石巨晶结构出露面. 早前的研究表明, 这些钾长石巨晶可能是岩浆演化早期饱和结晶形成 的堆晶(例如, Gilbert, 1906; Žák和Paterson,2005). 由式 (2) (4)的计算可以直观感受到, 直径 $10 \mathrm{~cm}$ 的巨晶较一 般粒径的钾长石晶体 (直径 $1 \mathrm{~mm}$ ) 沉降速度快约 10000 倍, 这与岩浆黏度下降3 4个数量级效果相当. 从理论 计算来看, 这些钾长石巨晶存在于岩浆房底部产生堆 积体的可能. 但随后有研究表明, 这些巨晶可能是在 底侵岩浆的热缓冲作用下形成的(Johnson和Glazner, 2010; Glazner和 Johnson, 2013). 我们认为, 对待类似 “卷轴状构造”、“钾长石巨晶堆晶”等结构的成因, 解 译时必须谨慎. 即便从物理角度看来, 用结晶分异对 这类结构进行解释较为合理, 但仍需更多的工作加以 佐证或排除. 在岩浆流动时, 如Glazner(2014)的计算所 暗含的, 在惯性运动方向上, 晶体与岩浆几乎不存相 对移动, 晶体的运动主要受控于岩浆的流动. 在中酸 性侵入体中, 此类结构更应该从非重力分异的角度进 行解析, 比如流动分异. 需要明确的是, 在紊乱流动的 岩浆中, 结晶矿物不能相对岩浆产生惯性位移, 这并
不意味着晶体与岩浆无法相对移动. 例如, 晶体在相 对静态的岩浆房中持续受重力作用, 给予一定的时间, 矿物仍可相对熔体发生显著位移; 而在流动的岩浆中, 矿物也可受剪切应力作用与熔体产生相对位移.

\section{2 静态晶䉼体的分离结晶作用一-Mush模型}

通过对不同结晶度下花岗质岩浆房的物理特性 及颗粒运动学分析可知, 在重力驱动下, 颗粒可通过 受阻沉降(hindered settling)、压实作用(compaction)等 机制与熔体分离. 被“抽离”的熔体可形成高硅花岗岩 或流纹岩, 而剩余堆晶(矿物晶体+粒间残余熔体)则 可能固结成为花岗岩(Gelman等, 2014). 这一结晶分异 过程就是近年来被越来越多研究者所关注的Mush模 型. 从2004年被提出至今, 不断有新的研究从物理过 程计算、计算机模拟、地球化学反演等角度验证、 理解这一地质过程(例如, Hildrelth, 2004; Bachmann和 Bergantz, 2004, 2008a, 2008b, 2008c; Gelman等, 2014; Lee等, 2015; Lee和Morton, 2015; Forni等, 2016).

Mush模型目前主要用于解释贫晶体流纹岩(Bachmann 和 Bergantz, 2004, 2008c; Hildreth, 2004; Streck 
和Grunder, 2008)、高硅花岗岩的成因(Lee和Morton, 2015; Lee等, 2015)及流纹岩-花岗岩的成因联系(Bachmann等, 2007; Lipman和 Bachmann, 2014; Keller等, 2015)等问题. Bachmann等(2007)对这一模型正反双 方的观点已有综述. 在此, 我们将侧重介绍该模型主 要的物理过程:

随着矿物结晶进行, 岩浆中晶体比例逐渐升高, 当 结晶度大于 0.4 时, 粒间中酸性熔体将逐渐演化为高硅 组分 (Bachmann和 Bergantz, 2004; Bachmann等, 2002). 当岩浆房结晶度增至 0.5 时, 岩浆对流将停止, 晶粥体 中的颗粒将形成渗透性网格. 通过受阻沉降、压实等 过程, 熔体可被“抽离”至岩浆房顶部, 形成高硅熔体 层(Bachmann和Bergantz, 2004; Bea, 2010). 若晶粥体 受到扰动, 高硅熔体层则可能上侵, 喷发形成流纹岩; 若赋存于地壳内部, 缓慢固结, 则可形成高硅花岗岩 (具高分异特征). 尔后, 当残留堆晶体结晶度达一定值 ( 0.7), 晶体与熔体分离过程停止, 岩浆将失去机械活 力而趋于固化(Bachmann和Bergantz, 2004; Bea, 2010; Hildreth, 2004)(图5). 所谓受阻沉降, 如前文计算结果 所示: 岩浆对流停止后, 晶体颗粒能以较低速率下沉. 压实是另一促使晶体-熔体分离的物理机制. 在矿物晶
体自重影响下, 晶粥体可自下而上将粒间熔体挤出(粒 度为2 $3 \mathrm{~mm}$ 的晶体, 通过压实作用产生 $500 \mathrm{~m}$ 尺度的 矿物-熔体分离需 $10^{4} \sim 10^{6} \mathrm{a}$ 完成, Bachmann和Bergantz, 2004).

假设有一个半径为 $2 \sim 3 \mathrm{~mm}$ 的石英或长石颗粒(密 度约为 $2600 \mathrm{~kg} \mathrm{~m}^{-3}$ ), 在岩浆房结晶度为 0.5 时开始记录 其下沉距离. 由于矿物颗粒结晶, 熔体的成分演化为 流纹质, 此时熔体密度 $2300 \mathrm{~kg} \mathrm{~m}^{-3}$. 计算可知, 颗粒沉 降 $500 \mathrm{~m}$ 所需时间约为 $10^{3} \sim 10^{4} \mathrm{a}$, 小于岩浆房演化寿命 $\left(10^{3} \sim 10^{5} \mathrm{a}\right.$, Cooper和Kent, 2014; Bachmann和 Bergantz, 2004; Lee等, 2015). 上述计算中, 结晶度被设定为一 常量. 但在实际过程中, 堆晶的结晶度将随熔体的抽 离而增大. 根据式(3)和(4)可知, 颗粒沉降速度将随之 减小. 假设矿物的沉降发生在大型岩浆房(面积大于 $10^{3} \mathrm{~km}^{2}$ ), 岩浆房厚 $5 \mathrm{~km}$. 若颗粒沉降 $0.5 \mathrm{~km}$, 岩浆房上 部即可汇聚 $500 \mathrm{~km}^{3}$ 流纹质熔体, 岩浆房结晶度由 0.5 上升为 0.556 . 对比图 2 可知, 以恒定的结晶度 0.5 进行 计算对结果影响不大, 时间尺度的量级仍为 $10^{3} \sim 10^{4} \mathrm{a}$. 如图6所示, 在流纹质岩浆黏度范围内 $\left(10^{4.5} \sim 10^{5.5} \mathrm{~Pa} \mathrm{~s}\right.$, Scaillet等, 1998), 不同黏度下, 结晶矿物均能在岩浆房 失活之前沉降足够的位移, 形成足量的高硅熔体层.

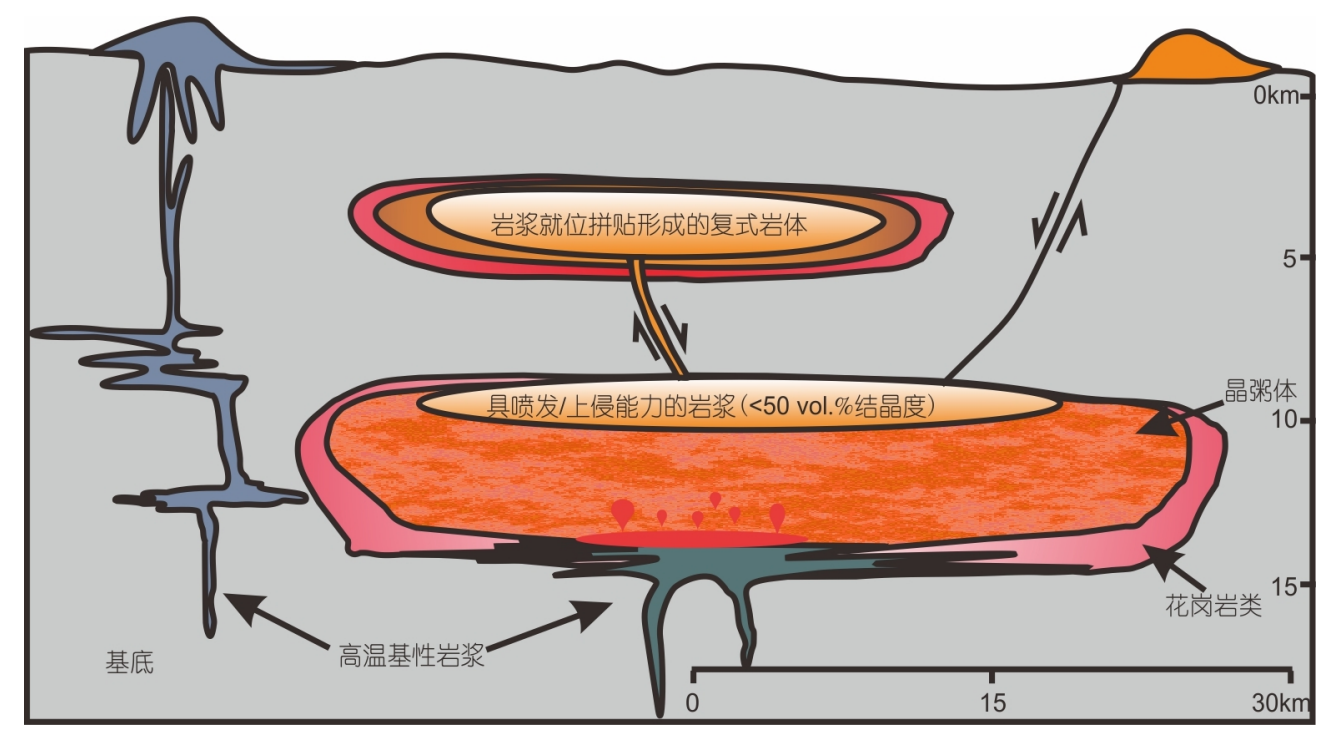

图 5 Mush模型图解

基于Bachmann和Bergantz(2008b)和Castro(2013)绘制. 当岩浆房达结晶阈值后, 岩浆房对流停止. 在静态的晶粥态岩浆房中, 由于缺少岩浆 对流的扰动, 在重力作用下通过受阻沉降和压实作用, 结晶矿物可与部分粒间熔体分离. 在岩浆房上部将形成贫晶体的高硅熔体层. 若具喷 发/上侵能力的岩浆喷发, 则可形成对应的流纹岩; 若熔体停留在岩浆房顶部或侵位于地壳, 则可形成具高分异特征的高硅花岗岩. 与之同 时, 高温岩浆底侵岩浆房是常见的地质现象. 高温岩浆底侵后, 固化的Mush将退晶粥化. 在接下来的过程中, 岩浆房将经历熔融、底侵岩 浆及围岩的混染; 随后冷却, 再次经历受阻沉降及压实作用. 此时形成的高硅熔体层成分会发生改变. 而热岩浆的底侵还可能触发顶部熔 体的上侵. 在不同的演化阶段触发上侵的岩浆, 成分上势必存在差异 


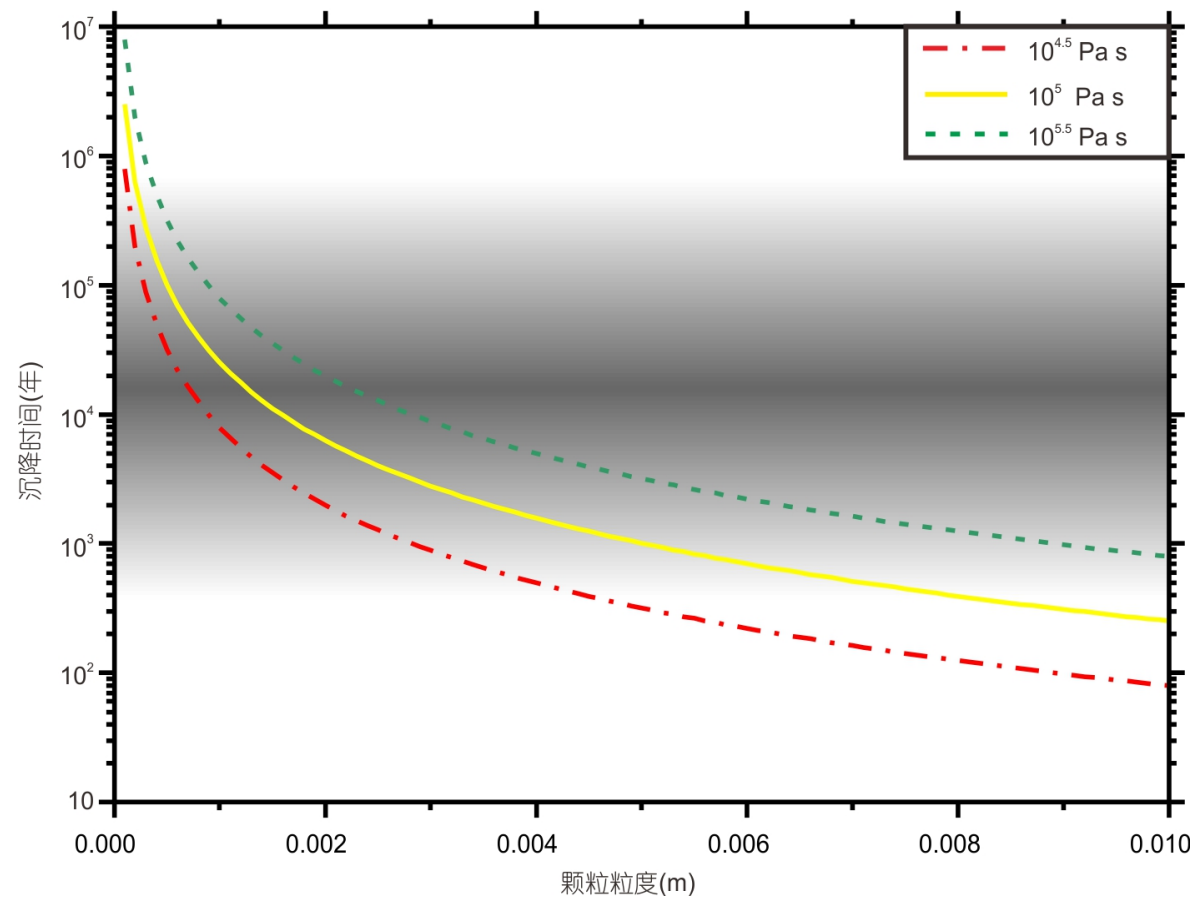

图 6 晶体-熔体分离 $500 \mathrm{~m}$ 尺度所需时间与颗粒粒度关系图

密度为 $2600 \mathrm{~kg} \mathrm{~m}^{-3}$ 的晶体(长石、石英)在硅酸岩晶粥体(晶体体积分数为 0.5 , 熔体密度 $2300 \mathrm{~kg} \mathrm{~m}^{-3}$ )中沉降 $500 \mathrm{~m}$ 时, 晶体半径与所需时间的关 系图, 给出了岩浆黏度分别为 $10^{4.5} 、 10^{5}$ 和 $10^{5.5} \mathrm{~Pa}$ s时的情况. 阴影部分为单期侵入的岩浆固结所需时间的范围

从物理理论计算角度来看, Mush模型可行.

物理理论计算为Mush模型提供了可行方案, 但这 一模型仍需更多的工作加以验证和完善. 我们在此想 特别强调, 在不同构造环境下, 晶粥体在演化过程中 晶体-熔体的分离程度可能有所差别. 如热岩浆的侵 入(Davidson和Tepley, 1997; Schmitt等, 2001; Tepley等, 2000; Peate等, 2008; Wark等, 2007; Forni等, 2016), 长 久来看可使晶体-熔体分离更加充分; 而断裂构造的 突然发育则会中断分离过程. 在不同条件下, Mush演 化程度会有所不同. 由此产生的堆晶花岗岩及顶部伴 生熔体层的地化特征也将存有差异(图5).

\section{3 成分变化的岩体: 岩浆房演化的产物?}

一些大型岩体存在 $10^{2} \sim 10^{4} \mathrm{~m}$ 的成分分带. 长期 以来, 这类分带的形成被认为是岩浆结晶分异(Bateman和 Chappell, 1979; Tindle 和 Pearce, 1981; Sisson 和 Moore, 1994) 或岩浆混合(Kistler等, 1986; Frost和Mahood, 1987)的结果. 这一研究思路的前提是岩体必须 由同一批岩浆演化而来. 即, 现在看到的岩体是由岩 浆房经历演化固结形成. 近来, 有关分带侵入体高精度
年代学的工作发现, 许多岩体的就位时间远大于通过 热模拟计算获得的岩浆房演化时间. 这一冲突为理解 一些大型侵入体不同部位矿物、成分和结构的变化, 提供了新的线索与思路(如Glazner等, 2004; Miller等, 2007; Scoates和Friedman, 2008; Scoates和Wall, 2015).

如美国内华达地区著名的Tuolumne岩体, 通过对 岩体进行细致综合的锆石U-Pb年代学工作发现, 该岩 套的锆石 $U-\mathrm{Pb}$ 年龄变化范围在 $85 \sim 95 \mathrm{Ma}$, 而被作为独 立填图单元的Half Dome花岗闪长岩, 其就位时间也 > 3Myr(Coleman等, 2004; Glazner等, 2004; Memeti等, 2010). 根据前述, $1 \mathrm{Myr}$ 基本上是单期侵入岩体中锆石 $\mathrm{U}-\mathrm{Pb}$ 年龄跨度的上限. 因此, Tuolumne岩体达 10Myr 的年龄间隔说明, 这些侵入体不可能由一期岩浆演化 形成. Coleman等(2004)认为该岩套的成分变化不会是 岩浆结晶分异的结果. 我们认为, 跨度如此之大的就 位时间, 实质上说明该岩体不是岩浆房固结的产物, 而 应该是多期岩浆拼合的集合体. 对于此类岩体, 运用 结晶分异讨论其成因并不具备讨论的前提. 目前研究 发现, 许多花岗岩基具有与 Tuolumne岩体相似的年代 学变化规律(Matzel等, 2006), 甚至很多岩体的年龄跨 
度超过10Myr, 比如中国华南广泛分布的印支期-燕山 期复式岩体(Ding等, 2015). Ding等(2015)应用活化的 Mush模型去解释这些复式岩体的成因. 即在经历后期 构造热事件作用后, 深部已晶粥化的岩浆房会发生退 晶䉼化; 活化产生的高硅熔体聚集到一定程度时, 由 于浮力的原因, 会穿透岩浆房而再次上侵(Burgisser和 Bergantz, 2011). 在此过程中, 活化产生的岩浆可能经 历岩浆混合、混染、Mush受阻沉降等演化过程, 从而 造成再次上涌的岩浆可能具有差异性的成分组成. 受 制于频繁的构造热事件, 这种退晶粥化过程可能持续 存在, 而相应的岩浆侵位事件也将以涌动或脉动的形 式出现, 在结晶年龄上也应与热事件耦合(图5). 因此, 对重要侵入体开展更为细致的年代学研究, 是花岗岩 研究中具有重要价值的工作方向(Coleman等, 2016), 或许是解决花岗岩侵位、演化等重大问题的突破口.

\section{4 华南富锂氟花岗岩由结晶分异形成垂向分带 的可能性}

对晶体颗粒沉降及岩浆房对流过程分析可知, 由 于花岗质岩浆中晶体沉降速度小, 且受前期岩浆对流 影响, 仅依靠晶体沉降产生岩体成分与矿物组合分带 的可能性微乎其微. 但在中国华南地区, 存在不少具 成分及矿物组合垂直分带特征的花岗岩类(图7), 如江 西雅山、湖南尖峰岭和广西栗木等岩体(Yin等, 1995; Zhu等, 2001). 且岩体结晶时间跨度 $<1 \mathrm{Myr}$ (如雅山岩 体中的中粗粒黑鳞云母-白云母花岗岩与锂云母花岗 岩的 ${ }^{206} \mathrm{~Pb} /{ }^{238} \mathrm{U}$ 年龄分别为(150.1 \pm 1.0$)$ 和(150.2 1 .4) Ma, 杨泽黎等, 2014), 在年龄误差范围内, 不能排除其为同 一期岩浆演化产物的可能. 这类岩体具有高的氟、磷 等挥发份及 $\mathrm{Li}^{+}$等变网离子含量, 促使熔体黏度与岩浆 固相线温度显著降低(Mysen等, 1981; 熊小林等, 1996; Xiong等,1999; Manning, 1981; Dingwell和Mysen, 1985; Sirbescu和Nabelek, 2003). 有关富锂氟花岗岩详尽系 统的地质学、岩石学、矿物学与地球化学特征, 吴福 元等(2017)已有论述总结. 对于富锂氟花岗岩的形成 机制, 前人的观点有热液交代成因及岩浆结晶分异成 因(朱金初等, 2002). 我们不拟就争论焦点进行铺述, 在此, 主要通过估算来阐明华南富锂氟花岗岩结晶分 异过程可能具有的特殊性. 即, 其岩浆就位后, 存在通 过重力分异形成成分及矿物组合垂向分带的可能.

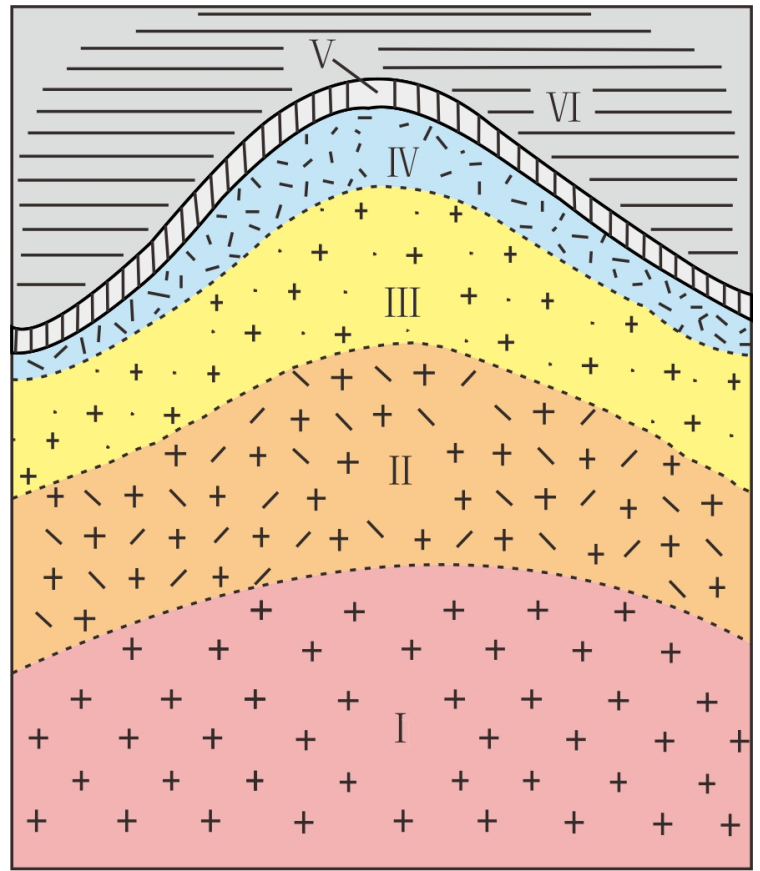

图 7 富锂氟花岗岩的垂直分带示意图

I, 二云母花岗岩带; II, 白云母花岗岩或浅色花岗岩带; III, 黄玉锂云母-钠长石花岗岩带; IV, 云英岩带; $V$, 钾长石伟晶岩壳和石英 壳; VI, 围岩. 据朱金初等(2002)修改

前人的实验及计算工作表明, 氟可有效降低岩浆 的黏度, 且随着温度降低, 富氟熔体相对于贫氟熔体黏 度下降程度将增大(Dingwell等, 1985; Mysen和 Virgo, 1985). Dingwell等(1985)测定了 $\mathrm{F}$ 对 $\mathrm{Na}_{2} \mathrm{O}-\mathrm{Al}_{2} \mathrm{O}_{3}-\mathrm{SiO}_{2}$ 体 系黏度的影响. 根据实验结果及对前人数据的计算 发现, 岩浆温度由 $1600^{\circ} \mathrm{C}$ 降至 $1000^{\circ} \mathrm{C}$ 过程中, 其体 系黏度均升高; 对钠长石熔体而言, 含 $5.8 \mathrm{wt} \% \mathrm{~F}$ 对比 $0 \mathrm{wt} \% \mathrm{~F}$ 的体系, 在 $1400^{\circ} \mathrm{C}$ 时其黏度下降约一个数量级, 在 $1000^{\circ} \mathrm{C}$ 条件下其黏度对比降低约 $2 \sim 3$ 个数量级. 由于 原作者没有进行更低温度的实验, 根据其黏度变化趋 势我们推断, 当岩浆温度降至 $1000^{\circ} \mathrm{C}$ 以下时, $5.8 \mathrm{wt} \% \mathrm{~F}$ 将使岩浆黏度下降至少 3 个数量级.

我们不妨将一般花岗质岩浆、F含量 $2 \mathrm{wt} \%$ 的花 岗质岩浆及玄武质岩浆的黏度及三类岩浆中晶体的 沉降速度做直观对比. 随着矿物结晶的进行, 花岗质 岩浆的熔体成分向流纹质不断演化, 持续富集水, 含 $4 \mathrm{wt} \% \mathrm{H}_{2} \mathrm{O}$ 的流纹质熔体黏度 $100^{4.5} \mathrm{~Pa} \mathrm{~s}$; 同理, 随着石 英、长石等贫 $\mathrm{F}$ 矿物的结晶, 富锂氟花岗质岩浆熔体 中F含量随之升高. 根据上述实验结果, 我们保守估计 
在 $800^{\circ} \mathrm{C}$ 左右条件下, 富锂氟花岗质岩浆的熔体较普 通花岗质岩浆熔体黏度下降约两个数量级(取 $10^{2.5} \mathrm{~Pa}$ $\mathrm{s}$ ); 而玄武质岩浆黏度在 10 100Pa s范围内(Murase和 McBirney, 1973), 我们取 $10^{1.5} \mathrm{~Pa}$ s以便对比计算. 假设 在结晶度为 0.5 的三个体系中均有一半径为 $2 \mathrm{~mm}$ 的晶 体颗粒, 其与周边熔体密度差均为 $300 \mathrm{~kg} \mathrm{~m}^{-3}$, 将参数 带入式(3) 可知, 普通花岗质岩浆中颗粒沉降速度为 $7.961 \times 10^{-9} \mathrm{~m} \mathrm{~s}^{-1}$ (即 $0.251 \mathrm{~m} \mathrm{a}^{-1}$ ), 富锂氟花岗质岩浆中的 晶体下降速度为 $7.961 \times 10^{-7} \mathrm{~m} \mathrm{~s}^{-1}$ (即 $25.1 \mathrm{~m} \mathrm{a}^{-1}$ ), 而玄武 质岩浆中的矿物沉降速度为 $7.961 \times 10^{-6} \mathrm{~m} \mathrm{~s}^{-1}$ (即 $251 \mathrm{~m}$ $\mathrm{a}^{-1}$ )(图8). 正是由于富含挥发份及 $\mathrm{Li}^{+}{ }^{+}$等变网离子, 富锂 氟花岗质岩浆的黏度可比一般花岗质岩浆小数个数 量级; 通过矿物沉降可在较小空间尺度内, 形成矿物及 成分的垂直分层(如雅山的黄玉锂云母花岗岩, $41.5 \mathrm{~m}$, 李洁和黄小龙, 2013). 但相比玄武质岩浆而言, 富锂 氟花岗质岩浆黏度依旧偏大, 晶体沉降速度仍然较小. 加之矿物共结序列的差异, 富锂氟花岗岩虽可见矿物 组合分层, 但不能如玄武质岩浆形成明显的矿物堆晶. 在一般岩浆体系中, $\mathrm{Zr} / \mathrm{Hf} 、 \mathrm{Nb} / \mathrm{Ta} 、 \mathrm{Y} / \mathrm{Ho}$ 等比值并无 明显变化(Green, 1995). 而富锂氟花岗岩可能因经历 强烈的结晶分异, 其全岩和锆石具较低的 $\mathrm{Zr} / \mathrm{Hf}$ 比值 (Bau, 1996; Breiter等, 2014). 当然, 这类地化特征也存 在由岩浆后期流体作用形成的可能(苏扣林等, 2015; 何俊杰等, 2015).

总的来说, 富锂氟花岗质岩浆黏度较一般花岗质 岩浆黏度要小数个数量级, 导致其中的颗粒具有更快 的沉降速度, 具有通过结晶分异形成垂向分层的可能.

\section{4 总结}

(1) 花岗质岩浆可发生结晶分异作用. 花岗质岩 浆非运移过程中的结晶分异, 可能更多地体现在流纹 岩、高硅花岗岩(具明显的高分异特征)和堆晶花岗岩 的形成以及大陆地壳成分的演化, 而对岩体成分变化 的贡献不大. 对于“Granites and Granites”(花岗岩的多 样性), 我们应该将更多的注意力放至Mush过程对上 侵物质成分的影响、岩浆运移过程中的分异及源区 反应的区别.

(2) 一般的花岗质岩浆难以通过重力分异形成垂 向分带, 而华南富锂氟花岗岩有通过结晶分异形成垂 向成分分带的可能. 富锂氟花岗岩的特殊性在于: 挥 发份及 $\mathrm{Li}^{+}{ }^{+}$等变网离子含量高, 较一般花岗质岩浆其黏

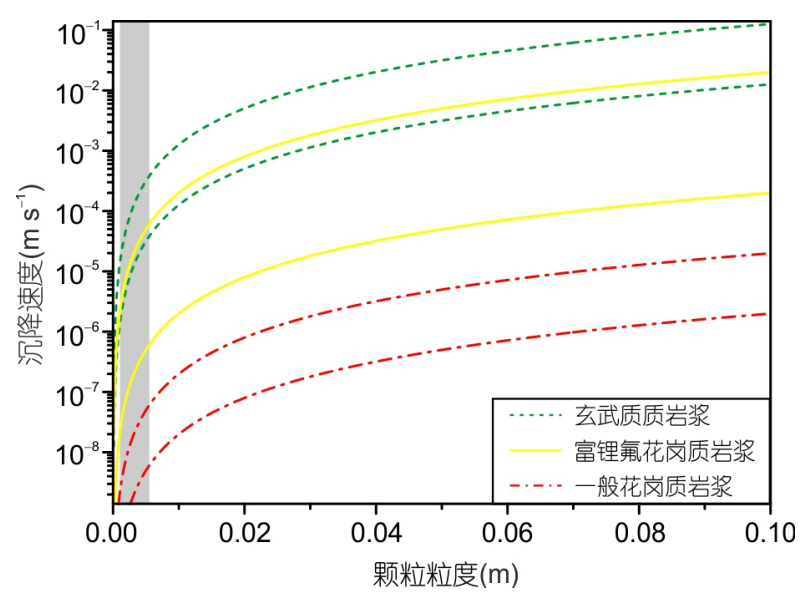

图 8 各岩浆体系中颗粒沉降速度与颗粒粒度关系图 同一颜色曲线所夹区间为对应体系中颗粒沉降速度的变化域. 成 分不同的岩浆体系的黏度存有显著差异, 矿物的沉降速度主要受体 系黏度影响. 当花岗质岩浆富锂氟等成分时, 黏度显著下降, 颗粒沉 降速度以数量级增加, 甚至可与玄武质岩浆体系中颗粒沉降速度重 合. 而在一般的花岗质岩浆中, 颗粒沉降速度明显要慢. 阴影部分表 示自然界多数晶体的粒径范围. 计算采用参数: 玄武质岩 $\Delta \rho$ 取 $600 \mathrm{~kg}$ $\mathrm{m}^{-3}$, 黏度变化范围为 10 100Pa s(Murase和McBirney, 1973); 一般花 岗质岩浆中 $\Delta \rho$ 取 $300 \mathrm{~kg} \mathrm{~m}^{-3}$, 黏度变化范围取 $10^{4.5} \sim 10^{5.5} \mathrm{~Pa}$ s(Scaillet等, 1998 ); 富锂氟花岗质岩浆 $\Delta \rho$ 取 $300 \mathrm{~kg} \mathrm{~m}^{-3}$, 黏度变化范围 $10^{1.5} \sim 10^{3.5} \mathrm{~Pa}$ s. 三类体系均研究结晶度 $c$ 均为 0.5 时的情况

度明显偏低, 其中的矿物颗粒沉降速度较快.

(3) 侵入体中, 在小尺度上表现出成分变化的结 构, 其成因需要进一步研究. 但可以明确的是, “假沉 积构造”形成过程中, 重力分异所起作用微弱; 而流动 分异不与物理分析矛盾, 可以进行, 但分异效应需要 更多的量化研究.

注意到近来火山系统研究中关于岩浆房存在状 态的争论(Coleman等, 2016; Lundstrom和Glazner, 2016; Wilson和Charlier, 2016; Clemens和Stevens, 2016), 我们 做如下补充: 如果岩浆是通过累积侵位机制侵位, 花 岗质岩浆初始时就以低熔体比例形态汇聚, 那么, 岩 浆房将直接由Mush阶段开始演化; 根据初始熔体比例 的差异, Mush演化的程度会有所不同. 这不影响文章 讨论的结果.

致谢在本文观点凝练和写作过程中, 吴福元研究员 多次提供了宝贵的意见和建议; 赵振华研究员、王强研 究员、马昌前教授和王孝否教授帮助进一步完善了本 文, 在此一并致谢. 


\section{参考文献}

何俊杰, 丁兴, 王玉荣, 孙卫东, 傅斌. 2015. 温度、浓度对流体中 氟钛络合物水解的影响: 对深部地质过程中钛元素活动的制约. 岩石学报, 31: 802-810

李洁, 黄小龙. 2013. 江西雅山花岗岩岩浆演化及其 $\mathrm{Ta}-\mathrm{Nb}$ 富集机制. 岩石学报, 29: 4311-4322

马昌前. 1987. 硅酸盐熔体的黏度、密度及其计算方法. 地质科技 情报, 6: 142-150

马昌前. 1989. 结晶分异作用的岩浆动力学条件. 地球科学一一 国地质大学学报, 14: 245-252

马昌前, 杨坤光, 唐仲华, 李增田. 1994. 花岗岩类岩浆动力学—理 论方法及鄂东花岗岩类例析. 武汉: 中国地质大学出版社. 260

苏扣林, 丁兴, 黄永贵, 郑小战, 吴凯, 胡永斌. 2015. 粤中早白严 世亚䯽山正长质杂岩体的成分分异及岩石成因. 岩石学报, 3: 829-845

吴福元, 李献华, 杨进辉, 郑永飞. 2007. 花岗岩成因研究的若干问 题. 岩石学报, 23: 1217-1238

吴福元, 刘小驰, 纪伟强, 王佳敏, 杨雷. 2017. 高分异花岗岩的识别与 研究. 中国科学: 地球科学, 47: 745-765, doi: 10.1360/N07201600139

熊小林, 朱金初, 饶冰. 1996. 黄玉云英岩成因的初步实验研究. 科 学通报, 41: 917-919

杨泽黎, 邱检生, 邢光福, 余明刚, 赵姣龙. 2014. 江西宜春雅山花岗 岩体的成因与演化及其对成矿的制约. 地质学报, 88: 850-868

张旗. 2012. 花岗质岩浆能够结晶分离和演化吗? 岩石矿物学杂志, 31: $252-260$

张旗, 潘国强, 李承东, 金惟俊, 贾秀勤. 2007. 花岗岩结晶分离作用 问题——关于花岗岩研究的思考之二. 岩石学报, 23: 1239-1251 朱金初, 饶冰, 熊小林, 李福春, 张佩华. 2002. 富锂氟含稀有矿化花 岗质岩石的对比和成因思考. 地球化学, 31: 141-152

Bachmann O, Bergantz G W. 2004. On the origin of crystal-poor rhyolites: Extracted from batholithic crystal mushes. J Petrol, 45: $1565-1582$

Bachmann O. 2011. 10 timescales associated with large silicic magma bodies. In: Dosseto A, Turner S, Van-Orman J, eds. Timescales of Magmatic Processes. Chichester: Blackwell Publishing. 212

Bachmann O, Bergantz G W. 2008a. Deciphering magma chamber dynamics from styles of compositional zoning in large silicic ash flow sheets. Rev Mineral Geochem, 69: 651-674

Bachmann O, Bergantz G W. 2008b. The magma reservoirs that feed supereruptions. Elements, 4: 17-21

Bachmann O, Bergantz G W. 2008c. Rhyolites and their source mushes across tectonic settings. J Petrol, 49: 2277-2285

Bachmann O, Dungan M A, Lipman P W. 2002. The fish canyon magma body, San Juan volcanic field, Colorado: Rejuvenation and eruption of an upper-crustal batholith. J Petrol, 43: 1469-1503

Bachmann O, Miller C F, de Silva S L. 2007. The volcanic-plutonic connection as a stage for understanding crustal magmatism. J Volcanol Geotherm Res, 167: 1-23

Barbey P. 2009. Layering and schlieren in granitoids: A record of interactions between magma emplacement, crystallization and deformation in growing plutons. Geol Belg, 12: 109-133

Barnea E, Mizrahi J. 1973. A generalized approach to the fluid dynamics of particulate systems. Chem Eng J, 5: 171-189

Barrière M. 1976. Flowage differentiation: Limitation of the Bagnold effect to the narrow intrusions. Contrib Mineral Petrol, 55: 139-145

Bateman P C. 1992. Plutonism in the central part of the Sierra Nevada batholith, California. U.S. Geological Survey Professional Paper, 1483: 186

Bateman P C, Chappell B W. 1979. Crystallization, fractionation, and solidification of the Tuolumne Intrusive Series, Yosemite National Park, California. Geol Soc Am Bull, 90: 465-482

Bau M. 1996. Controls on the fractionation of isovalent trace elements in magmatic and aqueous systems: Evidence from $\mathrm{Y} / \mathrm{Ho}, \mathrm{Zr} / \mathrm{Hf}$, and lanthanide tetrad effect. Contrib Mineral Petrol, 123: 323-333

Bea F. 2010. Crystallization dynamics of granite magma chambers in the absence of regional stress: Multiphysics modeling with natural examples. J Petrol, 51: 1541-1569

Becker G F. 1897. Fractional crystallization of rocks. Am J Sci, 22: $257-261$

Bergantz G W, Ni J. 1999. A numerical study of sedimentation by dripping instabilities in viscous fluids. Int $\mathrm{J}$ Multiphase Flow, 25: $307-320$

Bhattacharji S, Smith C H. 1964. Flowage differentiation. Science, 145: 150-153

Brandeis G, Marsh B D. 1989. The convective liquidus in a solidifying magma chamber: A fluid dynamic investigation. Nature, 339: 613-616

Breiter K, Lamarão C N, Borges R M K, Dall'Agnol R. 2014. Chemical characteristics of zircon from A-type granites and comparison to zircon of S-type granites. Lithos, 192-195: 208-225

Burgisser A, Bergantz G W. 2011. A rapid mechanism to remobilize and homogenize highly crystalline magma bodies. Nature, 471: 212-215

Campbell I H, Taylor S R. 1983. No water, no granites-No oceans, no continents. Geophys Res Lett, 10: 1061-1064

Castro A. 2013. Tonalite-granodiorite suites as cotectic systems: A review of experimental studies with applications to granitoid petrogenesis. Earth-Sci Rev, 124: 68-95

Chen C F, Turner J S. 1980. Crystallization in a double-diffusive system. J Geophys Res, 85: 2573-2593

Christiansen E H. 2005. Contrasting processes in silicic magma cham- 
bers: Evidence from very large volume ignimbrites. Geol Mag, 142: 669-681

Clarke D B, Clarke G K C. 1998. Layered granodiorites at Chebucto Head, South Mountain batholith, Nova Scotia. J Struct Geol, 20: 1305-1324

Clemens J D. 2015. Magmatic life at low Reynolds number: Comment. Geology, 43: e357-e357

Clemens J D, Helps P A, Stevens G. 2010. Chemical structure in granitic magmas-A signal from the source? Earth Environ Sci Trans R Soc Edinb, 100: 159-172

Clemens J D, Petford N. 1999. Granitic melt viscosity and silicic magma dynamics in contrasting tectonic settings. J Geol Soc, 156: 1057-1060

Clemens J D, Stevens G. 2012. What controls chemical variation in granitic magmas? Lithos, 134-135: 317-329

Clemens J D, Stevens G. 2016. Melt segregation and magma interactions during crustal melting: Breaking out of the matrix. Earth-Sci Rev, 160: $333-349$

Coleman D S, Gray W, Glazner A F. 2004. Rethinking the emplacement and evolution of zoned plutons: Geochronologic evidence for incremental assembly of the Tuolumne Intrusive Suite, California. Geology, 32: 433-436

Coleman D S, Mills R D, Zimmerer M J. 2016. The pace of plutonism. Elements, 12: 97-102

Cooper K M, Kent A J R. 2014. Rapid remobilization of magmatic crystals kept in cold storage. Nature, 506: 480-483

Davidson J P, Tepley F J. 1997. Recharge in volcanic systems: Evidence from isotope profiles of phenocrysts. Science, 275: 826-829

Davis R H, Acrivos A. 1985. Sedimentation of Noncolloidal particles at low Reynolds numbers. Annu Rev Fluid Mech, 17: 91-118

Ding X, Sun W D, Chen W F, Chen P R, Sun T, Sun S J, Lin C T, Chen F K. 2015. Multiple Mesozoic magma processes formed the 240-185 Ma composite Weishan pluton, South China: Evidence from geochronology, geochemistry, and Sr-Nd isotopes. Int Geol Rev, 57: 1189-1217

Dingwell D B, Mysen B O. 1985. Effects of water and fluorine on the viscosity of albite melt at high pressure: A preliminary investigation. Earth Planet Sci Lett, 74: 266-274

Dingwell D B, Scarfe C M, Cronin D J. 1985. The effect of fluorine on viscosities in the system $\mathrm{Na}_{2} \mathrm{O}-\mathrm{Al}_{2} \mathrm{O}_{3}-\mathrm{SiO}_{2}$ : Implications for phonolites, trachytes and rhyolites. Am Miner, (1-2): 80-87

Emeleus C H. 1963. Structural and petrographic observations on layered granites from southern Greenland. Mineral Soc Am Spec Pap . 22-29

Forni F, Bachmann O, Mollo S, De Astis G, Gelman S E, Ellis B S. 2016. The origin of a zoned ignimbrite: Insights into the Campanian Ignimbrite magma chamber (Campi Flegrei, Italy). Earth Planet Sci
Lett, 449: 259-271

Frost T P, Mahood G A. 1987. Field, chemical, and physical constraints on mafic-felsic magma interaction in the Lamarck Granodiorite, Sierra Nevada, California. Geol Soc Am Bull, 99: 272-291

Gaweda A, Szopa K. 2011. The origin of magmatic layering in the High Tatra granite, Central Western Carpathians - Implications for the formation of granitoid plutons. Earth Environ Sci Trans R Soc Edinb, 102: 129-144

Gelman S E, Deering C D, Bachmann O, Huber C, Gutiérrez F J. 2014. Identifying the crystal graveyards remaining after large silicic eruptions. Earth Planet Sci Lett, 403: 299-306

Gelman S E, Gutierrez F J, Bachmann O. 2013. On the longevity of large upper crustal silicic magma reservoirs. Geology, 41: 759-762

Gilbert G K. 1906. Gravitational assemblage in granite. Geol Soc Am Bull, 17: 321-328

Glazner A F. 2014. Magmatic life at low Reynolds number. Geology, 42: $935-938$

Glazner A F. 2015. Magmatic life at low Reynolds number: Reply. Geology, 43: e359-e359

Glazner A F, Bartley J M, Coleman D S, Gray W, Taylor R Z. 2004. Are plutons assembled over millions of years by amalgamation from small magma chambers? GSA Today, 14: 4

Glazner A F, Coleman D S, Bartley J M. 2008. The tenuous connection between high-silica rhyolites and granodiorite plutons. Geology, 36: 183-186

Glazner A F, Johnson B R. 2013. Late crystallization of K-feldspar and the paradox of megacrystic granites. Contrib Mineral Petrol, 166: 777-799

Green T H. 1995. Significance of $\mathrm{Nb} / \mathrm{Ta}$ as an indicator of geochemical processes in the crust-mantle system. Chem Geol, 120: 347-359

Grout F F. 1918. Two-phase convection in igneous magmas. J Geol, 26: 481-499

Gualda G A R, Ghiorso M S, Lemons R V, Carley T L. 2012. RhyoliteMELTS: A modified calibration of MELTS optimized for silica-rich, fluid-bearing magmatic systems. J Petrol, 53: 875-890

Gutiérrez F, Payacán I, Gelman S E, Bachmann O, Parada M A. 2013. Late-stage magma flow in a shallow felsic reservoir: Merging the anisotropy of magnetic susceptibility record with numerical simulations in La Gloria Pluton, central Chile. J Geophys Res-Solid Earth, 118: 1984-1998

Hildreth W. 1981. Gradients in Silicic magma chambers-Implications for lithospheric magmatism. J Geophys Res-Solid Earth, 86: 10153-10192

Hildreth W. 2004. Volcanological perspectives on Long Valley, Mammoth Mountain, and Mono Craters: Several contiguous but discrete systems. J Volcanol Geotherm Res, 136: 169-198 
Huber C, Bachmann O, Manga M. 2009. Homogenization processes in silicic magma chambers by stirring and mushification (latent heat buffering). Earth Planet Sci Lett, 283: 38-47

Jellinek A M, Kerr R C. 1999. Mixing and compositional stratification produced by natural convection: 2 . Applications to the differentiation of basaltic and silicic magma chambers and komatiite lava flows. J Geophys Res, 104: 7203-7218

Jellinek A M, Kerr R C, Griffiths R W. 1999. Mixing and compositional stratification produced by natural convection: 1. Experiments and their application to Earth's core and mantle. J Geophys Res, 104: 7183-7201

Johnson B R, Glazner A F. 2010. Formation of K-feldspar megacrysts in granodioritic plutons by thermal cycling and late-stage textural coarsening. Contrib Mineral Petrol, 159: 599-619

Keller C B, Schoene B, Barboni M, Samperton K M, Husson J M. 2015. Volcanic-plutonic parity and the differentiation of the continental crust. Nature, 523: 301-307

Kistler R W, Chappell B W, Peck D L, Bateman P C. 1986. Isotopic variation in the Tuolumne Intrusive Suite, central Sierra Nevada, California. Contrib Mineral Petrol, 94: 205-220

Koyaguchi T, Hallworth M A, Huppert H E, Sparks R S J. 1990. Sedimentation of particles from a convecting fluid. Nature, 343: 447-450

Lee C T A, Bachmann O. 2014. How important is the role of crystal fractionation in making intermediate magmas? Insights from $\mathrm{Zr}$ and P systematics. Earth Planet Sci Lett, 393: 266-274

Lee C T A, Morton D M. 2015. High silica granites: Terminal porosity and crystal settling in shallow magma chambers. Earth Planet Sci Lett, 409: 23-31

Lee C T A, Morton D M, Farner M J, Moitra P. 2015. Field and model constraints on silicic melt segregation by compaction/hindered settling: The role of water and its effect on latent heat release. Am Miner, 100: $1762-1777$

Lindsay J M, Schmitt A K, Trumbull R B, De S S L, Siebel W, Emmermann R. 2001. Magmatic evolution of the La Pacana caldera system, Central Andes, Chile: Compositional variation of two cogenetic, large-volume felsic ignimbrites. J Petrol, 42: 459-486

Lipman P W, Bachmann O. 2014. Ignimbrites to batholiths: Integrating perspectives from geological, geophysical, and geochronological data. Geosphere, 11: 705-743

Lundstrom C C, Glazner A F. 2016. Silicic magmatism and the volcanicplutonic connection. Elements, 12: 91-96

Manning D A C. 1981. The effect of fluorine on liquidus phase relationships in the system Qz-Ab-Or with excess water at $1 \mathrm{~kb}$. Contrib Mineral Petrol, 76: 206-215

Marsh B D. 1981. On the crystallinity, probability of occurrence, and rheology of lava and magma. Contrib Mineral Petrol, 78: 85-98
Marsh B D. 1989a. Magma chambers. Annu Rev Earth Planet Sci, 17: $439-472$

Marsh B D. 1989b. On convective style and vigor in sheet-like magma chambers. J Petrol, 30: 479-530

Marsh B D. 1996. Solidification fronts and magmatic evolution. Mineral Mag, 60: 5-40

Martin D, Nokes R. 1988. Crystal settling in a vigorously converting magma chamber. Nature, 332: 534-536

Matzel J E P, Bowring S A, Miller R B. 2006. Time scales of pluton construction at differing crustal levels: Examples from the Mount Stuart and Tenpeak intrusions, North Cascades, Washington. Geol Soc Am Bull, 118: 1412-1430

Maughan L L, Christiansen E H, Best M G, Grommé C S, Deino A L, Tingey D G. 2002. The Oligocene lund tuff, Great Basin, USA: A very large volume monotonous intermediate. J Volcanol Geotherm Res, 113: 129-157

McBirney A R. 1980. Mixing and unmixing of magmas. J Volcanol Geotherm Res, 7: 357-371

McBirney A R, Baker B H, Nilson R H. 1985. Liquid fractionation. Part I: Basic principles and experimental simulations. J Volcanol Geotherm Res, 24: 1-24

McKenzie D. 1984. The generation and compaction of partially molten rock. J Petrol, 25: 713-765

McKenzie D. 1985. The extraction of magma from the crust and mantle. Earth Planet Sci Lett, 74: 81-91

Memeti V, Paterson S, Matzel J, Mundil R, Okaya D. 2010. Magmatic lobes as "snapshots" of magma chamber growth and evolution in large, composite batholiths: An example from the Tuolumne intrusion, Sierra Nevada, California. Geol Soc Am Bull, 122: 1912-1931

Miller J S, Matzel J E P, Miller C F, Burgess S D, Miller R B. 2007. Zircon growth and recycling during the assembly of large, composite arc plutons. J Volcanol Geotherm Res, 167: 282-299

Murase T, McBirney A R. 1973. Properties of some common igneous rocks and their melts at high temperatures. Geol Soc Am Bull, 84: 3563-3592

Mysen B O, Ryerson F J, Virgo D. 1981. The structural role of phosphorus in silicate melts. Am Miner, 66: 106-117

Mysen B O, Virgo D. 1985. Structure and properties of fluorine-bearing aluminosilicate melts: The system $\mathrm{Na}_{2} \mathrm{O}-\mathrm{Al}_{2} \mathrm{O}_{3}-\mathrm{SiO}_{2}-\mathrm{F}$ at $1 \mathrm{~atm}$. Contrib Mineral Petrol, 91: 205-220

Oldenburg C M, Spera F J, Yuen D A, Sewell G. 1989. Dynamic mixing in magma bodies: Theory, simulations, and implications. J Geophys Res, 94: 9215-9236

Peate I U, Kent A J R, Baker J A, Menzies M A. 2008. Extreme geochemical heterogeneity in Afro-Arabian Oligocene tephras: Preserving fractional crystallization and mafic recharge processes in silicic 
magma chambers. Lithos, 102: 260-278

Petford N, Cruden A R, McCaffrey K J W, Vigneresse J L. 2000. Granite magma formation, transport and emplacement in the Earth's crust. Nature, 408: 669-673

Pitcher W S. 1997. The Nature and Origin of Granite. 2nd ed. London: Chapman \& Hall. 94, 387

Reid J B, Murray D P, Hermes O D, Steig E J. 1993. Fractional crystallization in granites of the Sierra Nevada: How important is it? Geology, 21: 587-590

Scaillet B, Holtz F, Pichavant M. 1998. Phase equilibrium constraints on the viscosity of silicic magmas: 1. Volcanic-plutonic comparison. J Geophys Res, 103: 27257-27266

Schmitt A K, de Silva S L, Trumbull R B, Emmermann R. 2001. Magma evolution in the Purico ignimbrite complex, northern Chile: Evidence for zoning of a dacitic magma by injection of rhyolitic melts following mafic recharge. Contrib Mineral Petrol, 140: 680-700

Scoates J S, Friedman R M. 2008. Precise age of the platiniferous Merensky Reef, Bushveld Complex, South Africa, by the U-Pb zircon chemical abrasion ID-TIMS technique. Econ Geol, 103: 465-471

Scoates J S, Wall C J. 2015. Geochronology of layered intrusions. In: Charlier B, Namur O, Latypov R, Tegner C, eds. Layered Intrusions. Dordrecht: Springer Netherlands. 3-74

Sirbescu M L C, Nabelek P I. 2003. Crustal melts below $400^{\circ} \mathrm{C}$. Geology, 31: 685

Sisson T W, Moore J G. 1994. Geologic Map of the Giant Forest Quadrangle, Tulare County, California. US Geological Survey

Smith T E. 1975. Layered granitic rocks at Chebucto Head, Halifax County, Nova Scotia. Can J Earth Sci, 12: 456-463

Solgadi F, Sawyer E W. 2008. Formation of igneous layering in granodiorite by gravity flow: A field, microstructure and geochemical study of the Tuolumne Intrusive Suite at Sawmill Canyon, California. J Petrol, 49: 2009-2042

Sparks R S J, Folkes C B, Humphreys M C S, Barfod D N, Clavero J, Sunagua M C, McNutt S R, Pritchard M E. 2008. Uturuncu volcano, Bolivia: Volcanic unrest due to mid-crustal magma intrusion. Am J Sci, 308: 727-769

Sparks R S J, Huppert H E, Turner J S, Sakuyama M, O’Hara M J. 1984. The fluid dynamics of evolving magma chambers. Philos Trans R Soc A-Math Phys Eng Sci, 310: 511-534

Stimac J A, Goff F, Wohletz K. 2001. Thermal modeling of the Clear Lake magmatic-hydrothermal system, California, USA. Geothermics, 30: $349-390$
Streck M J, Grunder A L. 2008. Phenocryst-poor rhyolites of bimodal, tholeiitic provinces: The Rattlesnake Tuff and implications for mush extraction models. Bull Volcanol, 70: 385-401

Street R L, Watters R J, Vennard J K. 1996. Elementary Fluid Mechanics. 7th ed. New York: John Wiley \& Sons. 233, 499

Taylor S R, McLennan S M. 1985. The Continental Crust: Its Composition and Evolution. Palo Alto: Blackwell Scientific Publication

Tepley F J, Davidson J P, Tilling R I, Arth J G. 2000. Magma mixing, recharge and eruption histories recorded in plagioclase phenocrysts from El Chichón Volcano, Mexico. J Petrol, 41: 1397-1411

Tindle A G, Pearce J A. 1981. Petrogenetic modelling of in situ fractional crystallization in the zoned Loch Doon pluton, Scotland. Contrib Mineral Petrol, 78: 196-207

Wager L R, Brown G M. 1968. Layered Igneous Rocks. San Francisco: WH Freeman. 587

Wallis G L. 1981. The geology of the wilsons promontory batholith, Victoria: A study of the composition, emplacement, and structure of an S-type granitoid. Dissertation for Master Degree. Melbourne: Monash University

Wark D A, Hildreth W, Spear F S, Cherniak D J, Watson E B. 2007. Pre-eruption recharge of the Bishop magma system. Geology, 35: 235-238

Whitney J A, Stormer J C. 1985. Mineralogy, petrology, and magmatic conditions from the fish canyon tuff, Central San Juan Volcanic Field, Colorado. J Petrol, 26: 726-762

Wilshire H G. 1969. Mineral layering in the Twin Lakes granodiorite, Colorado. Geol Soc Am Mem, 115: 235-262

Wilson C J N, Charlier B L A. 2016. The life and times of silicic volcanic systems. Elements, 12: 103-108

Xiong X L, Zhao Z H, Zhu J C, Rao B. 1999. Phase relations in albite granite- $\mathrm{H}_{2} \mathrm{O}-\mathrm{HF}$ system and their petrogenetic applications.. Geochem J, 33: 199-214

Yin L, Pollard P, Hu S X, Taylor R. 1995. Geologic and geochemical characteristics of the Yichun Ta-Nb-Li deposit, Jiangxi Province, South China. Econ Geol, 90: 577-585

Žák J, Paterson S R. 2005. Characteristics of internal contacts in the Tuolumne Batholith, central Sierra Nevada, California (USA): Implications for episodic emplacement and physical processes in a continental arc magma chamber. Geol Soc Am Bull, 117: 1242-1255

Zhu J C, Li R K, Li F C, Xiong X L, Zhou F Y, Huang X L. 2001. Topaz-albite granites and rare-metal mineralization in the Limu District, Guangxi Province, southeast China. Min Dep, 36: 393-405 